\title{
The facilitative glucose transporter and insulin action
}

\author{
Chan Y. Jung \\ The Biophysics Laboratory, VA Medical Center, and the Department of Biophysical \\ Sciences, State University of New York, Buffalo, NY 14215, U.S.A. \\ Accepted 9 December 1996
}

Abbreviations: GLUT, glucose transporter; GTBT, glucose transporter binding protein; GST, glutathione S-transferase; HAD, L-3-hydroxyacyl CoA dehydrogenase; TMH, transmembrane helix

\begin{abstract}
Cellular uptake of glucose is a tightly controlled process. It is mediated by a family of intrinsic membrane proteins (facilitated glucose transporters or GLUTs), and further regulated by metabolites and hormones. By far the most important GLUT regulation in human physiology is that by insulin, dysfunction of which may produce insulin resistance, a hallmark of diabetes mellitus and obesity. Six isoforms are now known in GLUT family. They differ in tissue-specific expression and regulation, yet share a common transmembrane topology showing a highly conserved transmembrane domain, and less conserved cytoplasmic and exoplasmic domains. Evidence indicates that the transmembrane domain accommodates a waterfilled glucose pathway (the catalytic domain). Little is known about the role of the exoplasmic domain. As for the cytoplasmic domain, a large body of evidence indicates its importance in tissue-specific regulation of GLUT function (the regulatory domain). This domain includes the $\mathrm{N}$-terminal segment, the large, central loop, and the Cterminal segment. Exactly how this domain participates in GLUT regulation is not known. An interesting possibility is that the regulation involves a specific cellular protein, which interacts with GLUT at the cytoplasmic domain and modulates the function. This putative, glucose transporter binding protein (GTBP) may be an enzyme, or a nonenzymatic adaptor or docking protein. Indeed, we have identified several cytosolic proteins that bind to the cytoplasmic domain of GLUT proteins; these include glyceraldehyde-3-phosphate dehydrogenase, glucokinase, GTBP70, GTBP85, GTBP28, and L-3-hydroxyacyl
\end{abstract}

CoA dehydrogenase. Interaction of GLUT protein with some of these GTPBs are functionally coupled. Whether any of these interactions is actually responsible for the insulin-induced GLUT regulation is yet to be determined.

\section{Introduction}

\section{Glucose transport in animal cells}

Virtually all living cells use glucose as a major energy and carbon source. The very first critical step of this glucose utilization is the entry (transport) of glucose into the cell across the plasma membrane diffusion barrier with a speed and selectivity compatible with metabolic requirement. Three distinct mechanisms are known to mediate this glucose entry (Silverman, 1991). The simplest of these is the mechanism known as facilitated diffusion or carrier-mediation (Jung, 1974). This mechanism is subserved by a family of intrinsic membrane proteins (the facilitated glucose transporter or GLUT, in short) (Pessin and Bell, 1992), and simply catalyses the transmembrane movement of glucose and other sugars with an enzyme-like stereoselectivity. It is an equilibrating system; it does not produce any glucose accumulation by itself. This particular glucose transport mechanism is found in all eukaryotic cells. Second mechanism is the so-called, sodium-glucose cotransport (recently reviewed by Koepsell and Spangenberg, 1994), which is subserved by another intrinsic membrane protein family known as SGLT family. This mechanism is different from the facilitated diffusion in that it catalyses an up-hill movement of glucose driven by a down-hill movement of sodium ion. This mechanism is operative in the apical membrane of epithelial cells as an essential step of transcellular uphill transport (absorption) of glucose. The third mechanism is known as group-translocation (Meadow et al., 1990). It is found only in bacteria, and subserved by the phosphoenolpyruvate phosphotransferase system (PTS). It catalyzes transmembrane movement of glucose and, at the same time, its phosphorylation, producing an intracellular accumulation of glucose phosphate. This review deals with the facilitated diffusion subserved by GLUT family only.

\section{Six isoforms of the facilitative glucose transporter family}

So far six distinct functional isoforms of GLUT family (GLUT1-5, and 7) have been identified by molecular cloning (Table I). They show a high amino acid 
Table I. The facilitated glucose transporter isoforms

\begin{tabular}{rll}
\hline Isoforms & \multicolumn{1}{c}{ Primary expression sites and other characteristics } & References \\
\hline GLUT1 & Human erythrocytes, brain endothelial cells, and transformed cells & (Mueckler et al. 1985) \\
2 & Liver, pancreatic beta cells, kidney and intestinal epithelia & (Asano et al., 1989) \\
3 & Neurons and placenta & (Kayano et al., 1988) \\
4 & Skeletal and heart muscles, adipocytes. Regulated by insulin. & (James et al., 1989) \\
5 & Small intestine and sperm cells. High affinity fructose transporter & (Kayano et al., 1990) \\
6 & No gene product & (Kayano et al., 1990) \\
7 & Liver. Mediates glucose exit from the endoplasmic reticulum & (Waddell et al., 1992) \\
\hline
\end{tabular}

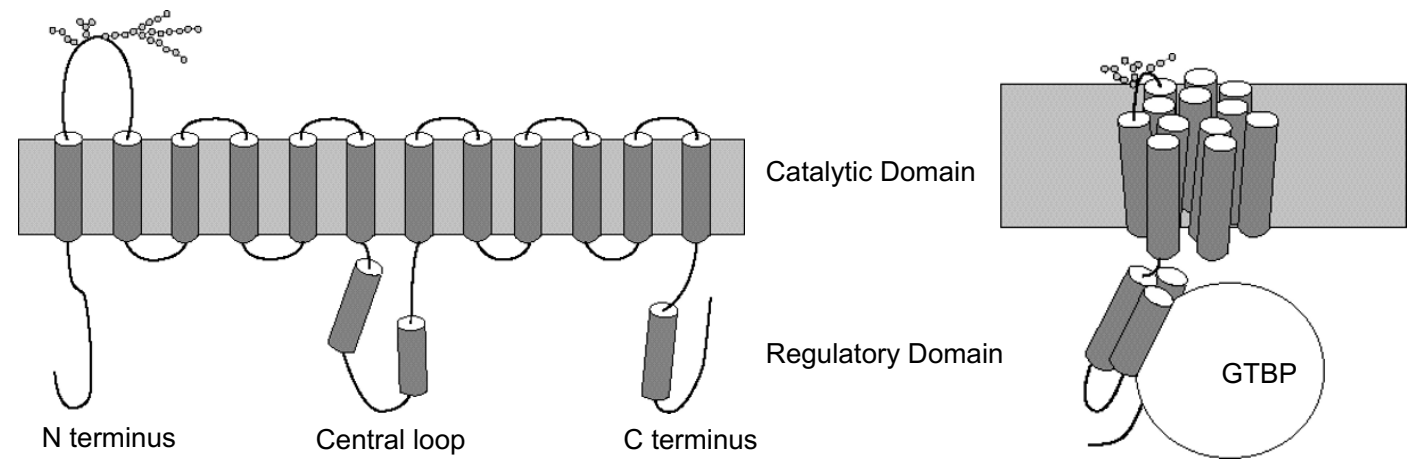

Figure 1. Left, GLUT1 transmem-brane topology and secondary structure. Right, functional domains, tertiary structure, and GTBP. A model.

sequence homology, yet differ significantly in tissuespecific expression, transport kinetics, substrate specificity, and, most importantly, in response to metabolic and hormonal regulation (Stephens and Pilch, 1995). Thus, insulin regulates GLUT4 function in muscles and adipocytes, a key control point for glucose homeostasis in humans and mammals (Birmbaum, 1992). The dysfunction of this regulation is known to produce the insulin resistance seen in diabetes and obesity (Garvey, 1992; James and Piper, 1994).

\section{Transmembrane topology common to all GLUT isoforms}

The cDNA-deduced amino acid sequences of six GLUT isoforms (Mueckler et al., 1985; Kayano et al., 1988; Asano et al., 1989; James et al., 1989; Kayano et al., 1990), together with the biochemical data obtained from purified GLUT1 protein (Kasahara and Hinkle, 1976; Baldwin et al., 1982; Cairns et al., 1987; Alvarez et al., 1987) indicate that all of these isoforms share a common transmembrane topology, having a large (about $50 \%$ of protein mass) transmembrane domain, with two grossly asymmetric nonmembrane domains, the cytoplasmic domain (about $35 \%$ of protein mass) and the exoplasmic domain (Figure 1). The trans- membrane domain is highly (97\%) conserved among the isoforms. Evidence indicates that this domain, made of twelve membrane-spanning alpha helices (transmembrane helices or TMHs 1-12) some of which line a water-filled glucose pathway (Mueckler et al, 1985, Jung et al., 1986), is similar for all isoforms (the catalytic domain). The cytoplasmic domain includes the amino and carboxyl termini, and the large central loop between TMHs 6 and 7 . The exoplasmic domain includes a fairly large, glycosylated loop between TMHs 1 and 2 . These nonmembrane domains show a significant amino acid sequence divergence, which suggests that they play a role in tissue-specific regulation of transporter function (the regulatory domain). Little data is available for the functional significance, if any, of the exoplasmic domain. For the cytoplasmic domain, an ample body of evidence indeed points to its importance in tissue-specific regulation of GLUT function, as will be discussed (vide infra). Exactly how, at the molecular level, the cytoplasmic domain participates in the regulation is not known. It is quite likely that specific cytosolic protein or proteins interact with GLUT protein at the cytoplasmic domain, thus modulates GLUT function in response to hormonal or metabolic signals (James et al., 1994). 


\section{How GLUT proteins transport glucose?}

\section{Transmembrane domain structure and glucose channel}

In contrast to the rapid progress witnessed in cloning these isoforms, the progress in understanding the structural basis of GLUT function and regulation has been disappointingly slow. GLUT1 of human erythrocytes is the only isoform available as a pure and functional protein (Rampal et al., 1986). Thus, the biochemical, biophysical, and structural informations available for this protein family are almost exclusively on this isoform. Circular dichroism measurements (Chin et al., 1987) have revealed that GLUT1 contains 70$80 \%$ alpha helices and some random coil and betaturns $(10 \%$ each), with no beta-structures. These data not only support the transmembrane helical structure predicted by hydropathy analysis (Mueckler et al., 1985), but also suggest the presence of a significant amount of alpha-helices in the nonmembrane domains, particularly in the cytoplasmic domain. Linear dichroism and Fourier transform infrared (FTIR) spectral measurements on oriented film (Chin et al., 1986) further indicate that all the twelve TMHs in GLUT1 are nearly perpendicular to the plane of the membrane, while nonmembrane (cytoplasmic) domain helices are quite flexible. Furthermore, hydrogen-tritium and hydrogen-deuterium exchange studies (Jung et al., 1986) offered a strong indication for the presence of an aqueous pore for glucose passage.

Based on these and other findings, we (Zheng et al., 1996) have recently proposed a working model for the structure of GLUT1 transmembrane domain, including the putative glucose channel. In this model, we emphasized the physical dimensional requirement of substrates and helical packing energy and macrodipole moments of the putative $12 \mathrm{TMHs}$, and predicted that TMHs 2, 5, 7, 8, and 11 form glucose channel. The model predicts discrete $\mathrm{TMH}$-lipid bilayer interface, TMH-transporter substrate interaction, as well as an interhelical proximity pattern for each TMH. This model is significantly different from the one previously proposed by Holman and his coworkers (Gould and Holman, 1993): They have proposed, primarily based on inhibitor binding studies, that only the $\mathrm{C}$-terminal half of the transmembrane domain (namely, TMHs 7-12) participates in glucose transport function. More recently, we (Lachaal et al., 1996) have shown that 3deoxy-3,3-azi-D-glucopyranose (3DAG), a photolyticallyreactive GLUT1 substrate, labels the $30-\mathrm{kDa}$ trypsin cleavage product containing TMHs 4 and 5 . This is a clear indication that both, in support of our model, $\mathrm{N}$ and C-terminal halves of the transmembrane domain line aqueous glucose channel.

Substrate transport is known to accompany significant conformational dynamics in GLUT1 protein (Rampal et al., 1987; Appleman and Lienhard, 1989). By studying the intrinsic fluorescence quenching of purified GLUT1 protein, we (Chin et al., 1992) have demonstrated dynamic interactions between Trp 388 and His 337, Trp 412 and Cys 347, and Trp 412 and Glu 380, depending on liganded state of GLUT1. We suggested that the kinky, bulky, and proline-rich cytoplasmic end of $\mathrm{TMH}-10$ may form a cam structure, and that this structure may undergo oscillational rotation upon binding and release of substrate, while cytochalasin B and forskolin block transport in part by preventing this oscillation.

\section{How GLUT proteins are regulated?}

\section{The three different levels of GLUT regulation}

By far the most important and well established mode of GLUT regulation is the stimulation of GLUT4 function by insulin in muscle and adipose cells (James and Piper, 1994). In the absence of insulin, the glucose transport activity (flux) in these two classical insulin target cells is very slow (by 100 fold) compared to those in noninsulin target cells such as brain, liver, or human erythrocytes, and it increases greatly upon insulin treatment, approaching to the speed that is seen in metabolically insulin-insensitive group of the cells (Silverman, 1991; Carruthers, 1990). Consistent with these long known facts is the finding that more than $95 \%$ of GLUT4 and $30-40 \%$ of GLUT1 in rat adipocytes are stored intracellularly, thus nonfunctional, and that insulin recruits these GLUTs from the storage pool to the plasma membrane (recruitment theory) (Cushman and Wardzala, 1980; Suzuki and Kono, 1980). In the former group of cells whose glucose transport function is not affected by insulin, practically all GLUTs are at the cell surface even in the absence of insulin.

The insulin-induced GLUT recruitment discussed above, however, does not always account for the entire insulin-induced glucose transport stimulation quantitatively, suggesting that insulin also increases the intrinsic activity of glucose transporters (ObermairKusser et al., 1990; Harrison et al., 1989). Recently, it also became clear that the intrinsic activity of GLUT1 is regulated in muscles, avian erythrocytes, and a cultured hepatocyte line in response to metabolic alteration such as reduced oxidative phosphorylation and hypoxia (Lawrence et al., 1990; Shetty et al., 1992).

Like other biologically important proteins, GLUT proteins are also regulated by alterations in the protein content available in cell. This cellular GLUT protein content, in turn, is determined by the rate of biosynthesis and the rate of degradation. Evidence indicates that 
both of these rates are regulated in muscle and adipocytes. Significant reduction in cellular GLUT4 or GLUT1 content occurs in experimentally induced diabetes in rats (Berger et al., 1989; Garvey et al., 1989; Moller and Flier, 1991). Reduction in cellular GLUT4 content in adipocytes of diabetic rats is accompanied by a compensatory reduction in the rate of GLUT4 degradation (Kim et al., 1994). In normal rat adipocytes, GLUT4 biosynthetically turns over with a half time of approximately $12 \mathrm{~h}$, which increases to $15 \mathrm{~h}$ or greater (each GLUT protein lives longer) when its synthesis was reduced in streptozotosin treated rats.

\section{The cytoplasmic domain may participate in GLUT regulation}

Evidence indicates that the cytoplasmic domain modulates GLUT protein catalytic activities: Lawrence Jr. et al. (1990) have shown that the phosphorylation of a C-terminal serine residue appears responsible for isoproterenol-induced inactivation of GLUT4 transport activity. Oka et al. (1990) have shown that a partial truncation of GLUT1 C-terminal sequence results in complete inactivation of GLUT1 transport activity. These findings suggest that the C-terminal cytoplasmic domain may modify the glucose pathway structure of the transporter. This modulation is likely to be regulated by a specific cellular protein through an interaction at this domain. Consistent with this notion is the experimentally supported suggestion that the cytoplasmic nonmembrane domain of GLUT1 contains a large amount of alpha-helices of flexible disposition (Chin et al., 1987).

Evidence also indicates that the cytoplasmic domain contains the putative molecular determinants for the constitutive and insulin-regulated GLUT targeting to the plasma membrane. Results of transfection of GLUT mutants and chimera in cultured cell lines (Mueckler, 1994; James et al., 1994; Verhey and Birnbaum, 1994; Verhey et al., 1993) support this contention. These studies show that primarily the C-terminus, and in part the central loop, determine constitutive propensity of GLUT1 and GLUT4 targeting to the plasma membrane. The $\mathrm{N}$-terminus, on the other hand, may be important for the retention of GLUT4 in its microsomal storage pool (James et al., 1994). These gene transfection experiments, however, failed to answer one important question, namely, which of these cytoplasmic subdomains is important in insulin-induced recruitment. This question can be answered most clearly if one can introduce each of mutated isoforms with a modified cytoplasmic domain as a purified protein into rat adipocytes, the best understood insulin target cell in terms of GLUT regulation, and study their behavior in response to insulin. We have shown that PEG-induced cell fusion protocol using rabbit erythrocyte ghosts makes such a reconstitution possible (unpublished data by Lee and Jung).

\section{Kinetic basis of insulin-induced GLUT4 recruitment}

Steady state kinetic measurements using an impermeable, photoreactive glucose analog have revealed that GLUT4 in rat adipocytes constantly recycles between the plasma membrane pool and its intracellular storage pool, with two discrete first-order rate constants, one for internalization $\left(k_{\text {in }}\right)$, and the other for externalization ( $\left.k_{\mathrm{ex}}\right)$ (Jhun et al., 1992). The study also revealed that insulin affects both rate constants, it reduced $k_{\text {in }}$ by 2.8 fold and increased $k_{\text {ex }}$ by 3.3 fold. These findings indicate that insulin-induced GLUT4 recruitment results from modulation of at least two, rate-limiting steps, namely, endocytosis and exocytosis, the biochemical basis of which may also be distinct, thus should be studied individually.

Okadaic acid, a well-known specific inhibitor of protein phosphatases 1 and 2A (PP1 and PP2A), stimulates glucose transport in adipocytes by inducing insulin-like recruitment of GLUT4 to the plasma membrane. By steady-state kinetic measurements, Jhun et al. (1995) have demonstrated that okadaic acid selectively increases GLUT4 externalization rate constant $\left(k_{\mathrm{ex}}\right)$ in rat adipocytes. Okadaic acid and insulin are not additive in the effect on GLUT4 recruitment; okadaic acid at higher concentrations interferes with the insulin effect. Based on these findings, they have suggested that insulin may also stimulate GLUT4 externalization by increasing the phosphorylation state of a specific serine/threonine phosphoprotein , possibly by a specific PP1 or PP2A. It is an interesting possibility that a phosphatase, or its associated protein, may interact with thus modulate GLUT4 externalization.

\section{How GLUTs receive insulin and metabolic signals}

Exactly how the cytoplasmic domain of a GLUT protein may modulate GLUT catalytic activity or participate in GLUT redistribution suggested above is an important open question. Protein-protein interaction is known to play a key role in many important cellular functions and regulations (Srere, 1987; Phizicky and Field, 1995; Myers Jr. and White, 1995), thus it is quite likely that a specific protein or proteins mediates the insulin signal by interacting with GLUT4 at its cytoplasmic domain. When insulin binds to its receptor, it activates the receptor tyrosine kinase activity by autophosphorylation (Kasuga et al., 1982). The activated tyrosine kinase of the insulin receptor causes tyrosyl-phosphorylation of IRS-1 (Rothenberg et al., 1991). The IRS-1 in turn interacts with and activates PI3-kinase (Backer et al., 
1992). This IRS-1-PI3-kinase interaction has been suggested to be part of the signal transduction pathway for insulin-induced GLUT4 redistribution (Kanai et al., 1993; Katagiri et al., 1996). The downstream elements in this signal pathway that link PI3 kinase and the related signal to GLUT4, however, are yet to be identified (Marcusohn et al., 1995). It is quite likely that this downstream pathway also involves a series of protein-protein interactions, including one that directly interacts with GLUT4 protein and modulates its subcellular redistribution or catalytic activity. The protein could be an enzyme such as a specific phosphokinase or phosphatase that covalently modifies GLUT4. It may be a non-enzymatic adaptor protein. To identify this putative GLUT4 regulatory protein, we have used several different assay methods and identified a number of glucose transporter binding proteins (GTBPs), as summarized below.

\section{GLUT binding proteins}

The possible association of GLUT proteins with other cellular proteins in vivo was first suggested by large target size, observed for glucose-sensitive cytochalasin B-binding activity in rat adipocytes (Jacobs et al., 1987), and subsequently supported by an in vitro demonstration of an ATP-modulated association of glyceraldehyde-3-phosphate dehydrogenase (GAPDH) with purified GLUT1 (Lachaal et al., 1990). More recently, the apparent requirement of GLUT2 expression in pancreatic beta cells for the insulin secretory response to ambient glucose (Unger, 1991) has been shown to be best explained by assuming an isoform-specific interaction of GLUT2 with glucokinase (Lachaal et al., 1993), again emphasizing the importance of a protein-protein interaction. Indeed, purified bacterial glucokinase (GK) binds to GLUT1 purified from human erythrocytes in vitro (Lachaal and Jung, 1993). This binding was abolished if GLUT1 was digested with trypsin, indicating that glucokinase binds to the cytoplasmic domain of GLUT1. The binding was stimulated by ADP and glucose 6-phosphate, but not by ATP and D-glucose. The binding caused no change in GLUT1 function, but it increased glucokinase enzymatic activity. A similar interaction may occur between GLUT2 and glucokinase and generate glucose signal for insulin secretion in pancreatic beta cells.

Using glutathione S-transferase (GST)-fusion protein technology, Liu et al. (1995) have identified a 70-kDa cytosolic protein (GTBP70) in rat adipocytes that binds to the cytoplasmic domains of GLUT1, GLUT2 and GLUT4. The binding showed little isoform-specificity, but was significantly subdomain-specific; it bound to the C-terminal domain and the central loop, but not to the $\mathrm{N}$-terminal domain of GLUT4. The binding was inhibited by ATP in a dose-dependent manner, but not by ADP and AMP-PCP. GTBP70 was not metabolically labeled with ${ }^{32} \mathrm{P}$, suggesting that it is not a phosphoprotein. The binding, however, was little affected by insulin treatment. The role, if any, of GTBP70 in glucose transporter regulation in adipocytes is yet to be demonstrated.

Also using GST-fusion proteins, Shi et al. (1995) have identified two GTBPs (GTBPs 28, and 85) in Clone 9 cell cytosol, which may be involved in azideinduced GLUT1 modulation. A brief (1-2 h) exposure to azide is known to increase glucose uptake in Clone 9 cells, without increasing the plasma membrane level of GLUT1, the only GLUT isoform expressed in these cells (Shetty et al., 1992). The glucose-displaceable, cytochalasin B-binding activity was barely detectable in Clone-9 cells, but increased greatly after a $2-\mathrm{h}$ incubation with azide. Furthermore, the cytochalasin B binding activity of purified GLUT1 was inactivated by azide-treated Clone 9 cell cytosol, but not by untreated cytosol. These findings strongly suggest the presence of a cytosolic protein in Clone 9 cells that inactivates GLUT1, and an azide-induced metabolic alteration releases GLUT1 from this inhibitory interaction. Shi et al. (1995) showed that GTBP28 is a 28-kDa cytosolic protein, and binds to the cytoplasmic domain of GLUT1 and GLUT4. They also demonstrated that GTBP85 is a 85-kDa phosphoprotein, and binds to GLUT4, but not to GLUT1, and only in azide-treated Clone 9 cytosol, but not in control cytosol. The roles, if any, of these proteins in azide-induced modulation of GLUT1 substratebinding activity are to be demonstrated.

More recently, we used a yeast-based, two hybrid system (Yang et al., 1992; Guarente, 1993) to search for GTBPs. Screening a human skeletal muscle library with GLUT4 C-terminus as bait, revealed one clone (C10) which encodes an interactive protein (unpublished observation by Shi, Y.W., Samuel, S.J., Lee, W., Yu, C.H., Zhang, W., Lachaal, M. and Jung, C.Y.). The interaction was not an artifact of two hybrid system, as C-10 by itself, GAL4bd alone, lamin, or Glut1C was incapable of activating beta-galactosidase activity. The deduced amino acid sequence of this protein (YP10) was found to be $89 \%$ identical, and $92 \%$ similar, to the well-known, pig heart mitochondrial L-3-hydroxyacyl CoA dehydrogenase (HAD). An in vitro binding assay using GST-YP10 fusion protein and synthetic peptides confirmed that YP10 binds to GLUT4C, but not to GLUT1C. Most significantly, recombinant YP10 in vitro showed the dehydrogenase activity of HAD, which was inhibited by GLUT4C peptide, but not by GLUT1C peptide. YP10 crossreacts with anti-HAD antisera in immunoblot. Subcellular distribution of anti-HAD antisera immunoreactivity revealed that YP10 and/or HAD are/is associated not only with mitochondria, but also with the plasma membrane, but is vertually absent 
in microsomes in rat adipocytes. This suggests that YP10 is an isoform of HAD that interacts with GLUT4 selectively at the plasma membrane, and is thereby down-regulated. The physiological significance of this interaction is not known. It may be a apart of GLUT4 regulation mechanism involving protein acylation.

\section{Other potential GTBPs which may participate in modulation of GLUT4 recycling}

There are a few interesting GTBP candidates that may be involved in regulation of GLUT4 recycling. The GLUT4 recruitment in rat adipocytes in response to insulin is controlled at two discrete rate-limiting steps (represented by the first-order rate constants, $k_{\text {in }}$ and $\left.k_{\text {ex }}\right)$. This suggests that separate mechanisms for endocytosis and exocytosis, where two distinct GTBPs, $\mathrm{X}$ and $\mathrm{Y}$, modulate $k_{\text {in }}$ and $k_{\mathrm{ex}}$, respectively. $\mathrm{X}$ could be a clathrin or AP2-like protein if not clathrin or AP-2 itself (Moore et al., 1987). Y could be NSF and/or SNAP like protein(s) if not NSF or SNAP itself (Warren, 1990). They may also be specific GTP-binding proteins (Keller et al., 1991; Baldwin et al., 1991; Douset and Tuana, 1991) or specific kinases (Del Vecchio and Pilch, 1991; Clancy and Czech, 1990). N-ethylmaleimide (0.05-1 $\mathrm{mM}$ ) inhibits the insulin-stimulated (but not the basal) glucose transport, and arrests insulin-induced (but not basal) GLUT4 translocation (data to be published). This suggested us that a $\mathrm{N}$-ethylmaleimide-sensitive factor (NSF) may be required for GLUT translocation. NSF and SNAP may be involved in GLUT4 externalization, most likely acting at the step of IRGTV fusion to plasma membrane. It is also known that $\mathrm{N}$-ethylmaleimide at low concentrations ( $0.1 \mathrm{mM}$ or above) interacts with a membrane protein and effectively inhibits LDL-receptor internalization in fibroblasts (Lin et al., 1991).

\section{Concluding remarks}

Understanding at the molecular level how insulin causes GLUT4 recruitment in normal cells would give an insight to the identification of the nature of the defect underlying the insulin resistance. Although exact nature is not known, it becomes increasingly evident that the cytoplasmic domain of GLUT proteins is involved in this insulin-induced GLUT recruitment by interacting with a specific cellular protein or proteins. These putative regulatory proteins are expected to modulate one or both of the two rate-limiting steps in GLUT4 recycling. The identification, cloning, and characterization of this GLUT4 regulatory GTBP will contribute significantly to the elucidation of the molecular mechanisms by which insulin regulates glucose transport in normal muscles and adipose tissue.

Since the two hybrid screening and the in vitro binding assay using GST-fusion protein use GLUT4 cytoplasmic domain segments rather than whole protein, they may miss the GTBP-GLUT4 interaction responsible for the insulin action, if the cytoplasmic segments do not fold into a native conformation. Although not likely, the interaction of X with GLUT may not involve the cytoplasmic domain. Finally, the putative protein X may not exist; GLUT4 targeting may be regulated by a non-protein, secondary messenger such as lipid. Even in these unlikely situations, the systematic search as proposed would certainly uncover a number of GTBPs that may be important for other aspects of the GLUT function and regulation. These include regulation at the levels of catalytic activity, and of metabolic turnover. Alternatively, some GTBPs identified may be an important enzyme whose function is regulated via its interaction with GLUT4.

\section{Acknowledgements}

I am grateful to many of my coworkers, past and present, for their excellent work and many insightful discussions. Supported in part by grants from National Institutes of Health, American Heart Association, and by the Medical Research of Buffalo VA Medical Center.

\section{Literature references}

Alvarerez, J., Lee, D. C., Baldwin, S. A. and Chapman, D. (1987) Fourier transform infrared spectroscopic study of the structure and conformational changes of the human erythrocyte glucose transporter. J. Biol. Chem. 262: 3502-3509

Appleman, J. R. and Lienhard, G. E. (1989) Kinetics of the purified glucose transporter Direct measurement of the rates of interconversion of transporter conformers. Biochemistry 28: 8221-8226

Asano, T., Takaku, F. and Oka, Y. (1989) The nucleotide sequence of cDNA for a mouse liver-type glucose transport protein. Nucleic Acid Res. 17: 6386-92

Backer, J. M., Myers, M. G. Jr., Shoelson, S. E., Chin, D. J., Sun, X. J., Miralpeix, M., Hu, P., Margolis, B., Skolnik, E. Y. and Schlessinger, J. (1992) Phosphatidylinositol 3'kinase is activated by association with IRS-1 during insulin stimulation. EMBO J. 11 : 3469-3479

Baldwin, S. A., Baldwin, J. M. and Lienhard, G. E. (1982) Monosaccha-ride transporter of the human erythrocyte. Characterization of an improved preparation. Biochemistry 21: $3836-3842$

Baldwin, G., Hohman, R., Charron, M. J. and Lodish, H. F. (1991) Insulin and nonhydrolyzable GTP analogs induce translocation of GLUT4 to the plasma membrane in a toxin-permeabilized rat adipose cells. J. Biol. Chem. 266: 4037-4040

Birnbaum, M. J. (1992) The insulin-sensitive glucose transporter. Int. Rev. Cytol. 137A: 239-297

Cairns, M. P., Alvarez, J., Panico, M., Gibbs, A. F., Morris, H. R., Chapman, D. and Baldwin, S. A. (1987) Investigation of the structure and function of the human erythrocyte glucose transporter by proteolytic dissection. Biochim. Biophys. Acta 905: 295-310.

Carruthers, A. (1990) Facilitated diffusion of glucose. Physiol. Rev. 70:1135-1176.

Chin, J. J., Jhun, B. H. and Jung, C. Y. (1992) Structural basis of human erythrocyte glucose transporter function: intrinsic fluorescence studies. Biochemistry 31: 1945- 
1951

Chin, J. J., Jung, E. K. Y. and Jung, C. Y. (1986) Structural basis of human erythrocyte glucose transporter function in reconstituted vesicles: a-helix orientation. J. Biol. Chem. 261: 7101-7104

Chin, J. J., Jung, E. K. Y. and Jung, C. Y. (1987) Structural basis of human erythrocyte glucose transporter function in reconstituted vesicles. Circular dichroism studies. Proc. Natl. Acad. Sci. U.S.A. 84: 4113-4116

Clancy, B. M. and Czech, M. P. (1990) Hexose transport stimulation and membrane redistribution of glucose transporter isoforms in response to cholera toxin, dibutyryl cyclic AMP, and insulin in 3T3LI adipoctes. J. Biol. Chem. 265: 12434-12443

Cushman, S. W. and Wardzala, L. J. (1980) Potential mechanism of insulin action on glucose transport in the isolated rat adipose cells: apparent translocation of intracellular transport systems to the plasma membrane. J. Biol. Chem. 255: 47584762

Del Vecchio, R. L. and Pilch, P. F. (1991) Phosphatidylinositol 4-kinase is a component of glucose transporter (GLUT4)-containing vesicles. J. Biol. Chem. 266: 13278-13283

Doucet, J. P. and Tuana, B. S. (1991) Identification of low molecular weight GTPbinding proteins and their sites of interaction in subcellular fractions from skeletal muscle. J. Biol. Chem. 266: 17613-17620

Garvey, W. T. (1992) Glucose transport and NIDDM. Diabetes Care 15: 396-417.

Gould, G. W. and Holman, G. D. (1993) The glucose transporter family: structure, function and tissue-specific expression. Biochem. J. 295: 329-341

Gould, G. W. and Lienhard, G. E. (1989) Expression of a functional glucose transporter in Xenopus oocytes. Biochemistry 28: 9447-9452

Guarente, L. (1993) Strategies for the identification of interesting proteins. Proc. Natl. Acad. Sci.U.S.A. 90: 1639-1641

Haring, H. (1989) Further evidence for a two-step model of glucose transport regulation. Biochem. J. 261: 699705

Harrison, S. A., Buxton, J. M. and Czech, M. P. (1991) Suppressed intrinsic catalytic activity of GLUTI glucose transporters in insulin- sensitive 3T3-LI adipocytes. Proc. Natl. Acad. Sci. U.S.A. 88: 7839-7843

Ismail-Beigi, F. (1993) Metabolic regulation of glucose transport. J. Membrane Biol. 135: $1-10$

Jacobs, D. B., Berenski, C. J., Spangler, R. A. and Jung, C. Y. (1987) Radiation inactivation target size of rat adipocyte glucose transporters in the plasma membrane and intracellular pools. J. Biol. Chem. 262: 8084-8087

James, D. E., Strube, M. and Mueckler, M. (1989) Molecular cloning and characterization of an insulin-regulatable glucose transporter. Nature 338: 83-87

James, D. E. and Piper, R. C. (1994) Insulin resistance, diabetes, and the insulinregulated trafficking of GLUT4. J. Cell Biol. 126: 1123-1126

James, D. E., Piper, R. C. and Slot, J. W. (1994) Insulin stimulation of GLUT4 translocation: a model for regulated recycling. Trends Cell Biol. 4: 120-126

Jhun, B. H, Rampal, A. L., Liu, H. Z., Lachaal, M. and Jung, C. Y. (1992) Effects of insulin on steady state kinetics of GLUT4 subcellular distribution in rat adipocytes: evidence of constitutive GLUT4 recycling. J. Biol. Chem. 267: 17710-17715

Jung, C. Y. (1974) The carrier-mediated glucose transport across human red cell membranes. In The Red Blood Cell (Surgenor, D. M., ed.), Vol 2, p. 705, Academic Press, New York

Jung, E. K. Y., Chin, J. J. and Jung, C. Y. (1986) Structural basis of human erythrocyte glucose transporter function in reconstituted system: hydrogen exchange. J. Biol. Chem. 261: $9155-9160$

Kasahara, M., Hinkle, P. C. (1976) Reconstitution of D-glucose transport catalyzed by a protein fraction from human erythrocytes in sonicated liposomes. Proc. Natl. Acad. Sci. U.S.A. $73: 396-400$
Kanai, F., Ito, K., Todaka, M., Hayashi, H., Kamohara, S., Ishii, K., Okada, T., Hazeki, O., Ui, M. and Ebina, Y. (1993) Insulin-stimulated GLUT4 translocation is relevant to the phosphorylation of IRS-1 and the activity of PI3-kinase. Biochem. Biophys. Res. Commun. 195: 762-768

Kasuga, M., Zick, Y., Blith, D. L., Karlsson, F. A., Haring, H. U. and Kahn, C. R. (1982) Insulin stimulation of phosphorylation of the beta subunit of the insulin receptor Formation of both phosphoserine and phosphotyrosine. J. Biol. Chem. 257: 9891-9894

Katagiri, H., Asano, T., Ishihara, H., Inugkai, K., Shibasaki, Y., Kikuchi, M., Yazaki, Y. and Oka, Y. (1996) J. Biol. Chem. 271: 16987-16990

Kayano, T., Burant, C. F., Fukumoto, H., Gould, G. W., Fan, Y. S., Eddy, R. L., Byers, M. G., Shows, T. B., Seino, S. and Bell, G. I. (1990) Human facilitative glucose transporters: isolation, functional characterization, and gene localization of CDNAs encoding an isoform (GLUT5) expressed in small intestine, kidney, muscle, adipose tissue and an unusual glucose transporter pseudogene-like sequence (GLUT6). J. Biol. Chem. 265: 13276-13282

Kayano, T. Fukumoto, R. L. Eddy, A. L., Fan, Y.-S., Byers, M. G., Shows, T. B. and Bell, G. I. (1988) Evidence for a family of human glucose transporter-like proteins. Sequence and gene localization of a protein expressed in fetal skeletal muscle and other tissues. J. Biol. Chem. 263: 15245-15251

Keller, S. R., Kitagawa, K., Aebersold, R., Lienhard, G. E. and Garner, C. W. (1991) Isolation and characterization of the 160,000-Da phosphotyrosyl protein, a putative participant in insulin signaling. J. Biol. Chem. 266: 12817-12820

Kim, S. S., Bae, J. W. and Jung, C. Y. (1994) GLUT4 degradation rate: reduction in rat adipocytes in fasting and streptozotocin-induced diabetes. Am. J. Physiol. 267: E132E139

Koepsell, H. and Spangenberg, J. (1994) Function and presumed molecular structure of $\mathrm{Na}^{+}$-D-glucose transport systems. J. Membrane Biol. 138: 1-11

Lachaal, M., Berenski, C. J., Kim, J. and Jung, C. Y. (1990) An ATP-modulated specific association of glyceraldehyde-3-phosphate dehydrogenases with human erythrocyte glucose transporter. J. Biol. Chem. 265: 15449-15454

Lachaal, M., Jhun, B. H, Liu, H., Manka, M., Spangler, R. A. and Jung, C. Y. (1995) Okadaic acid increases GLUT4 externalization rate constant in rat adipocytes. J. Biol. Chem. 270: 3938-3943

Lachaal M. and Jung C. Y. (1993) Interaction of facilitated glucose transporter with glucokinase and its modulation by glucose-6-phosphate. J. Cell Physiol. 156: 326-332

Lachaal M., Rampal A. L., Lee W. and Jung C. Y. (1996) Trans-membrane glucose channel: Affinity labeling with a transportable D-glucose diazirine. J. Biol. Chem. 271: 5225-30

Lachaal, M., Spangler,R. A. and Jung. C. Y. (1993) High $K_{m}$ of GLUT2 alone does not explain its role in insulin secretion. Am. J. Physiol. 265: E914-E919

Lin, H. C., Moor, M. S., Sanan, D. A. and Anderson, G. W. (1991) Reconstitution of clathrin-coated pit budding from plasma membranes. J. Cell Biol. 114: 881-891

Liu, H. Z, Xiong, S. H., Samuel, S. J., Shi, Y. W., Lachaal, M. and Jung, C. Y. (1995) ATP sensitive binding of a $70 \mathrm{kDa}$ cytosolic protein to glucose transporter in rat adipocytes. J. Biol. Chem. 270: 7869-7875

Lawrence, J. C. Jr., Hiken, J. F. and James, D. E. (1990) Stimulation of glucose transport and glucose transporter phosphorylation by okadaic acid in rat adipocytes. $J$. Biol. Chem. 265: 19768-19776

Marcusohn, J., Isakoff, S. J., Rose, E., Symons, M. and Skolnik, E. Y. (1995) The GTPbinding protein Rac does not couple PI3-kinase to insulin-stimulated glucose transport in adipocytes. Curr. Biol. 5: 1296-1302

Meadow, N. D., Fox, D. K. and Roseman, S. (1990). Annu. Rev. Biochem. 59: 497-542

Moller, D. E. and Flier, J. S. (1991) Insulin resistance: mechanisms, syndromes and implications. New Engl. J. Med. 325: 983-948 
Moore, M. S., Mahaffey, D. T., Brodsky, F. M. and Anderson, R. G. W. (1987) Assembly of clathrin-coated pits onto purified plasma membranes. Science 338 : 558563

Mueckler, M. (1994) Facilitative glucose transporters. Eur. J. Biochem. 219: 713-725

Mueckler, M., Caruso, C., Baldwin, S. A., Panico, M., Blench, I., Morris, H. R., Allard, W. J., Lienhard, G. E. and Lodish, H. F. (1985) Sequence and structure of a human glucose transporter. Science 229: 941-945

Myers, Jr., M. G. and White, M. F. (1995) New frontiers in insulin receptor substrate signaling. Trends Endocrinol. Metab. 6: 209-215

Obermaier-Kusser, B., Muhlbacher, C., Mushack, J., Seffer, E., Ermel, B., Machicao, F., Schmidt, F., Oka, Y, Asano, T., Shibasaki, Y., Lin J. L., Tsukuda, K., Katagiri, H., Akanuma, Y. and Takaku, F. (1990) C-Terminal truncated glucose transporter is locked into an inward-facing form without transport activity. Nature 345: 550-553

Pessin, J. E. and Bell, G. I. (1992) Mammalian facilitative glucose transporter family: Structure and molecular regulation. Annu. Rev. Physiol. 54: 911-930

Phizicky, E. M. and Fields, S. (1995) Protein-protein interactions: methods for detection and analysis. Microbiol. Rev. 59: 94-123

Rampal, A. L., Jung, E. K. Y., Chin, J. J., Deziel, M. R., Pinkofsky, H. B. and Jung, C. $Y$. (1986) Further characterization and chemical purity assessment of the human erythrocyte glucose transporter preparation. Biochim. Biophys. Acta 959: 1235-1242

Rothenberg, P. L., Lane, W. S., Karasik, A., Baker, J., White, M. and Kahn, C. R. (1991) Purification and partial sequence analysis of pp185, the major cellular substrate of the insulin receptor tyrosine kinase. J. Biol. Chem. 266: 8302-8311

Shetty, M., Loeb, J. N. and Ismail-Beigi, F. (1992) Enhancement of glucose transport in response to inhibition of oxidative metabolism: pre- and post-translational mechanisms. Am. J. Physiol. 262 (Cell Physiol. 31): C527-C532

Shi, Y. W., Liu, H., Vanderburg, G., Samuel, S. J., Ismail-Beigi, F. and Jung, C. Y. (1995) Modulation of GLUT1 intrinsic activity in Clone 9 cells by inhibition of oxidative phosphorylation. J. Biol. Chem. 270: 21772-21778

Silverman, M. (1991) Structure and function of hexose transporters. Annu. Rev. Biochem. 60: 757-794

Srere, P. A. (1987) Complexes of sequential metabolic enzymes. Annu. Rev. Biochem. 56: $89-124$

Suzuki, K. and Kono, T. (1980) Evidence that insulin cause translocation of glucose transport activity to the plasma membrane from an intracellular storage site. Proc. Natl. Acad. Sci. U.S.A. 77 : 2542-2545

Verhey, K. J. and Birnbaum, M. J. (1994) A leu-leu sequence is essential for $\mathrm{COOH}$ terminal targeting signal of GLUT4 glucose transporter in fibroblasts. J. Biol. Chem. 269: 2353-2356
Verhey, K. J., Hausdorff, S. F. and Birnbaum, M. J. (1993) Identification of the carboxy terminus as important for the isoforrn-specific subcellular targeting of glucose transporter proteins. J. Cell Biol. 123: 137-147

Waddell, I. D., Zomerschoe, A. G., Voice, M. W. and Burchell, A. (1992) Cloning and expression of a hepatic microsomal glucose transport protein. Comparison with liver plasma membrane glucose transport protein GLUT2. Biochem. J. 286: 173-177

Warren, G. (1990) Intracellular transport: vesicular consumption. Nature 345: 382-383

Yang, X., Hubbard, E. J. A. and Carlson, M. (1992) A protein kinase substrate identified by the two-hybrid system. Science 257: 680-682

Zheng, H., Rampal, A. L., Parthasarathy, R. and Jung, C. Y. (1996) Proposed structure of putative glucose channel in GLUT1 facilitative glucose transporter. Biophys. J. 70: $14-21$ 